\title{
The rigid-flexible robotic manipulator: Nonlinear control and state estimation considering a different mathematical model for estimation
}

\author{
André Fenili \\ Universidade Federal do ABC (UFABC), CECS/Aerospace Engineering Av. dos Estados, 5001 - Bloco B, Sala \\ 936 - Santo André/SP - CEP 09210 580, Brazil \\ Tel.: +55 1149960134 ; E-mail: andre.fenili@ufabc.edu.br
}

\begin{abstract}
In this paper the author investigates the angular position and vibration control of a nonlinear rigid-flexible two link robotic manipulator considering fast angular maneuvers. The nonlinear control technique named State-Dependent Riccati Equation (SDRE) is used here to achieve these aims. In a more realistic approach, it is considered that some states can be measured and some states cannot be measured. The states not measured are estimated in order to be used for the SDRE control. These states are all the angular velocities and the velocity of deformation of the flexible link. A SDRE-based estimator is used here. Not only different initial conditions between the system to be controlled (here named "real" system) and the estimator but also a different mathematical model is considered as the estimation model in order to verify the limitations of the proposed state estimation and control techniques. The mathematical model that emulates the real system to be controlled considers two modes expansion and the estimation model considers only one mode expansion. The results for the different approaches are compared and discussed.
\end{abstract}

Keywords: Nonlinear systems, nonlinear state estimation, nonlinear control, robotic manipulators, flexible structures, SDRE

\section{Introduction}

With the increasing demand for precise high-speed operation and lightweight mechanisms for space missions, it was no longer adequate to treat certain links in a manipulator as rigid [17]. Expanding this idea, a rigid-flexible two-link manipulator can be lighter and faster than a rigid-rigid one and can cover a wider workspace using less energy. Lightweight mechanical structures are expected to improve the performance of robotic manipulators, which also often have low payload-to-arm mass ratios. However, such manipulators with flexible parts exhibit undesirable vibrations which might limit their performance [13]. The investigation of these systems can also be considered as a starting point for more complicated multi-link flexible or rigid-flexible manipulators. This kind of system finds many applications in industry and mainly in aerospace engineering.

Several methods have been developed for handling with the design of state observers and control algorithms for the kind of nonlinear system investigated in this work. The State-Dependent Riccati Equation (or simply SDRE) method, developed over the past several years, is one such method.

The SDRE control technique for nonlinear regulator problems has become well-known within the control community [5]. This method has been successfully employed in a considerable number of mathematical and real applications $[3,8,9,11,12,16]$.

A good survey of the SDRE design technique can be found in [4]. The SDRE based estimator is derived by constructing the dual of the well-known SDRE nonlinear regulator control design technique [5]. 


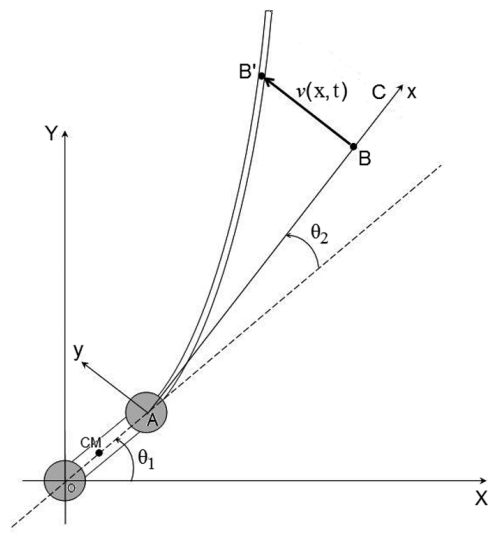

Fig. 1. The slewing flexible beam system.

\section{The geometric model and governing equations of motion}

Figure 1 presents the geometric model of the dynamic system investigated in this work. This system comprises a rigid link connected to a flexible link, each one of them driven by a DC motor. In this figure, the inertial axis is represented by XY, the moving axis (attached to the flexible link and rotating with it) is represented by $x y$, the beam deflection (as a space-time variable) is represented by $v(x, t)$ and the angular displacement angles are given by $\theta_{1}$ and $\theta_{2}$.

The governing equations of motion are obtained through the lagrangian formalism. To apply the Lagrange's equations one needs to know the kinetic energy stored in the rigid link and the kinetic and potential (strain) energies stored in the beam-like flexible link during its time evolution.

The total kinetic energy, $T$, of the system depicted in Fig. 1 is given by:

$$
\begin{aligned}
T= & \frac{1}{2}\left(I_{1}+m_{1} L_{c 1}^{2}\right) \dot{\theta}_{1}^{2}+\frac{1}{2} \int_{0}^{L_{2}} \rho A_{2}\left\{\left[\dot{\nu}+x\left(\dot{\theta}_{1}+\dot{\theta}_{2}\right)\right]^{2}+\left[\nu\left(\dot{\theta}_{1}+\dot{\theta}_{2}\right)\right]^{2}\right. \\
& \left.+2 L_{1} \dot{\theta}_{1}\left(\dot{\theta}_{1}+\dot{\theta}_{2}\right)\left(x \cos \theta_{2}-\nu \sin \theta_{2}\right)+2 L_{1} \dot{\nu}_{1} \cos \theta_{2}+L_{1}^{2} \dot{\theta}_{1}^{2}\right\} d x
\end{aligned}
$$

where $I_{1}$ represents the moment of inertia of the cross-section area of the rigid link, $m_{1}$ represents the mass of the rigid link, $L_{c 1}$ represents the location of the center of mass of the rigid link, $\rho$ represents the density of the material of the rigid link, $A_{2}$ represents the cross-section area of the flexible link and $L_{1}$ represents the length of the rigid link.

The strain energy of the system depicted in Fig. 1 is given by:

$$
V=\frac{1}{2} \int_{0}^{L_{2}} E I_{2} \nu^{\prime \prime 2} d x
$$

In Eq. (2), $E$ represents the Young's modulus of the material of the flexible link, $I_{2}$ represents the moment of inertia of the cross-section area of the flexible link and $L_{2}$ represents the length of the flexible link.

The Lagrangian is given by:

$$
L=T-V
$$

One assumes here linear curvature for the flexible link $[6,7,15]$. The Lagrangian is discretized considering the expansion given by:

$$
v(x, t)=\sum_{i=1}^{n} \Phi_{i}(x) q_{i}(t)
$$


where $n$ represents the number of normal modes, $\Phi_{i}(x)$ represents each one of the normal modes of the flexible structure and $q_{i}(t)$ represents time dependant elastic coordinates.

Inertia-free normal modes $[1,10]$ are considered. The discretized Lagrangian considering $n=2$ is given by:

$$
\begin{aligned}
L= & \frac{1}{2}\left(I_{1}+m_{1} L_{c 1}^{2}+\frac{\rho A_{2} L_{1}^{2} L_{2}}{2}+\frac{\rho A_{2} L_{2}^{3}}{6}\right) \dot{\theta}_{1}^{2}+\frac{\rho A_{2}}{2} \sum_{i=1}^{2} \sum_{j=1}^{2}\left(\int_{0}^{L_{2}} \Phi_{i} \Phi_{j} d x\right) \dot{q}_{i} \dot{q}_{j}+\rho A_{2}\left(\dot{\theta}_{1}+\dot{\theta}_{2}\right) \\
& \sum_{i=1}^{2}\left(\int_{0}^{L_{2}} x \Phi_{i} d x\right) \dot{q}_{i}+\frac{\rho A_{2} L_{2}^{3}}{3} \dot{\theta}_{1} \dot{\theta}_{2}+\frac{\rho A_{2} L_{2}^{3}}{6} \dot{\theta}_{2}^{2}+\frac{\rho A_{2}}{2}\left(\dot{\theta}_{1}+\dot{\theta}_{2}\right)^{2} \sum_{i=1}^{2} \sum_{j=1}^{2}\left(\int_{0}^{L_{2}} \Phi_{i} \Phi_{j} d x\right) q_{i} q_{j} \\
& +\frac{\rho A_{2} L_{1} L_{2}^{2}}{2} \dot{\theta}_{1}^{2} \cos \theta_{2}+\frac{\rho A_{2} L_{1} L_{2}^{2}}{2} \dot{\theta}_{1} \dot{\theta}_{2} \cos \theta_{2}-\rho A_{2} L_{1} \dot{\theta}_{1}^{2} \sin \theta_{2} \sum_{i=1}^{2}\left(\int_{0}^{L_{2}} \Phi_{i} d x\right) q_{i} \\
& -\rho A_{2} L_{1} \dot{\theta}_{1} \dot{\theta}_{2} \sin \theta_{2} \sum_{i=1}^{2}\left(\int_{0}^{L_{2}} \Phi_{i} d x\right) q_{i}+\rho A_{2} L_{1} \dot{\theta}_{1} \cos \theta_{2} \sum_{i=1}^{2}\left(\int_{0}^{L_{2}} \Phi_{i} d x\right) \dot{q}_{i} \\
& -\frac{E I_{2}}{2} \sum_{i=1}^{2} \sum_{j=1}^{2}\left(\int_{0}^{L_{2}} \Phi_{i}^{\prime \prime} \Phi_{j}^{\prime \prime} d x\right) q_{i} q_{j}
\end{aligned}
$$

Expanding the summations, this Lagrangian can be rewriten as:

$$
\begin{aligned}
L= & \frac{1}{2}\left(I_{1}+m_{1} L_{c 1}^{2}+\frac{\rho A_{2} L_{1}^{2} L_{2}}{2}+\frac{\rho A_{2} L_{2}^{3}}{6}\right) \dot{\theta}_{1}^{2}+\frac{\rho A_{2}}{2}\left[\left(\int_{0}^{L_{2}} \Phi_{1}^{2} d x\right) \dot{q}_{1}^{2}+2\left(\int_{0}^{L_{2}} \Phi_{1} \Phi_{2} d x\right) \dot{q}_{1} \dot{q}_{2}\right. \\
& \left.+\left(\int_{0}^{L_{2}} \Phi_{2}^{2} d x\right) \dot{q}_{2}^{2}\right]+\rho A_{2}\left(\dot{\theta}_{1}+\dot{\theta}_{2}\right)\left(\int_{0}^{L_{2}} x \Phi_{1} d x\right) \dot{q}_{1}+\rho A_{2}\left(\dot{\theta}_{1}+\dot{\theta}_{2}\right)\left(\int_{0}^{L_{2}} x \Phi_{2} d x\right) \dot{q}_{2}+\frac{\rho A_{2} L_{2}^{3}}{3} \dot{\theta}_{1} \dot{\theta}_{2} \\
& +\frac{\rho A_{2} L_{2}^{3}}{6} \dot{\theta}_{2}^{2}+\frac{\rho A_{2}}{2}\left(\dot{\theta}_{1}+\dot{\theta}_{2}\right)^{2}\left[\left(\int_{0}^{L_{2}} \Phi_{1}^{2} d x\right) q_{1}^{2}+2\left(\int_{0}^{L_{2}} \Phi_{1} \Phi_{2} d x\right) q_{1} q_{2}+\left(\int_{0}^{L_{2}} \Phi_{2}^{2} d x\right) q_{2}^{2}\right] \\
& +\frac{\rho A_{2} L_{1} L_{2}^{2}}{2} \dot{\theta}_{1}^{2} \cos \theta_{2}+\frac{\rho A_{2} L_{1} L_{2}^{2} \dot{\theta}_{1} \dot{\theta}_{2} \cos \theta_{2}-\rho A_{2} L_{1} \dot{\theta}_{1}^{2} \sin \theta_{2}\left(\int_{0}^{L_{2}} \Phi_{1} d x\right) q_{1}-\rho A_{2} L_{1} \dot{\theta}_{1}^{2} \sin \theta_{2}}{2} \\
& \left(\int_{0}^{L_{2}} \Phi_{2} d x\right) q_{2}-\rho A_{2} L_{1} \dot{\theta}_{1} \dot{\theta}_{2} \sin \theta_{2}\left(\int_{0}^{L_{2}} \Phi_{1} d x\right) q_{1}-\rho A_{2} L_{1} \dot{\theta}_{1} \dot{\theta}_{2} \sin \theta_{2}\left(\int_{0}^{L_{2}} \Phi_{2} d x\right) q_{2} \\
& +\rho A_{2} L_{1} \dot{\theta}_{1} \cos \theta_{2}\left(\int_{0}^{L_{2}} \Phi_{1} d x\right) \dot{q}_{1}+\rho A_{2} L_{1} \dot{\theta}_{1} \cos \theta_{2}\left(\int_{0}^{L_{2}} \Phi_{2} d x\right) \dot{q}_{2} \\
& -\frac{E I_{2}}{2}\left[\left(\int_{0}^{L_{2}} \Phi_{1}^{\prime \prime} \Phi_{1}^{\prime \prime} d x\right) q_{1}^{2}+2\left(\int_{0}^{L_{2}} \Phi_{1}^{\prime \prime} \Phi_{2}^{\prime \prime} d x\right) q_{1} q_{2}+\left(\int_{0}^{L_{2}} \Phi_{2}^{\prime \prime} \Phi_{2}^{\prime \prime} d x\right) q_{2}^{2}\right]
\end{aligned}
$$

In Eq. (5), $q_{1}$ represents time dependant elastic coordinates of the first flexural mode and $q_{2}$ represents time dependant elastic coordinates of the second flexural mode. 
The free vibration mode shapes of continuous systems satisfy the orthogonality conditions:

$$
\int_{0}^{L} \Phi_{1} \Phi_{2} d x=0
$$

and

$$
\int_{0}^{L} \Phi_{1}^{\prime \prime} \Phi_{2}^{\prime \prime} d x=0
$$

Therefore, Eq. (5) can be rewriten as:

$$
\begin{aligned}
L= & \frac{I_{T}}{2} \dot{\theta}_{1}^{2}+\frac{\eta_{1}}{2} \dot{\theta}_{1}^{2} q_{1}^{2}+\frac{\eta_{2}}{2} \dot{\theta}_{1}^{2} q_{2}^{2}+\eta_{1} \dot{q}_{1}^{2}+\eta_{2} \dot{q}_{2}^{2}+\eta_{3} \dot{\theta}_{1} \dot{q}_{1}+\eta_{3} \dot{\theta}_{2} \dot{q}_{1}+\eta_{4} \dot{\theta}_{1} \dot{q}_{2}+\eta_{4} \dot{\theta}_{2} \dot{q}_{2} \\
& +\eta_{5} \dot{\theta}_{1} \dot{\theta}_{2}+\frac{\eta_{5}}{2} \dot{\theta}_{2}^{2}+2 \eta_{1} \dot{\theta}_{1} \dot{\theta}_{2} q_{1}^{2}+\eta_{1} \dot{\theta}_{2}^{2} q_{1}^{2}+2 \eta_{2} \dot{\theta}_{1} \dot{\theta}_{2} q_{2}^{2}+\eta_{2} \dot{\theta}_{2}^{2} q_{2}^{2}+\eta_{6} \dot{\theta}_{1}^{2} \cos \theta_{2}+\eta_{6} \dot{\theta}_{1} \dot{\theta}_{2} \cos \theta_{2} \\
& -\eta_{7} \dot{\theta}_{1}^{2} q_{1} \sin \theta_{2}-\eta_{8} \dot{\theta}_{1}^{2} q_{2} \sin \theta_{2}-\eta_{7} \dot{\theta}_{1} \dot{\theta}_{2} q_{1} \sin \theta_{2}-\eta_{8} \dot{\theta}_{1} \dot{\theta}_{2} q_{2} \sin \theta_{2}+\eta_{7} \dot{\theta}_{1} \dot{q}_{1} \cos \theta_{2}+\eta_{8} \dot{\theta}_{1} \dot{q}_{2} \cos \theta_{2} \\
& -\eta_{9} \omega_{1}^{2} q_{1}^{2}-\eta_{10} \omega_{2}^{2} q_{2}^{2}
\end{aligned}
$$

where $\omega_{1}$ is the natural frequency of the first flexural mode and $\omega_{2}$ is the natural frequency of the second flexural mode and:

$$
\begin{aligned}
& \alpha_{1}=\int_{0}^{L_{2}} \Phi_{1} d x \quad \alpha_{2}=\int_{0}^{L_{2}} \Phi_{2} d x \quad \alpha_{3}=\int_{0}^{L_{2}} x \Phi_{1} d x \quad \alpha_{4}=\int_{0}^{L_{2}} x \Phi_{2} d x \quad \gamma_{1}=\int_{0}^{L_{2}} \Phi_{1}^{2} d x \quad \gamma_{2}=\int_{0}^{L_{2}} \Phi_{2}^{2} d x \\
& I_{T}=I_{1}+m_{1} L_{c 1}^{2}+\frac{\rho A_{2} L_{1}^{2} L_{2}}{2}+\frac{\rho A_{2} L_{2}^{3}}{6} \quad \eta_{1}=\frac{\rho A_{2} \gamma_{1}}{2} \quad \eta_{2}=\frac{\rho A_{2} \gamma_{2}}{2} \quad \eta_{3}=\rho A_{2} \alpha_{3} \quad \eta_{4}=\rho A_{2} \alpha_{4} \\
& \eta_{5}=\frac{\rho A_{2} L_{2}^{3}}{3} \quad \eta_{6}=\frac{\rho A_{2} L_{1} L_{2}^{2}}{2} \quad \eta_{7}=\rho A_{2} L_{1} \alpha_{1} \quad \eta_{8}=\rho A_{2} L_{1} \alpha_{2} \quad \eta_{9}=\frac{\rho A_{2} \gamma_{1}}{2} \quad \eta_{10}=\frac{\rho A_{2} \gamma_{2}}{2} \\
& \Phi_{1}^{\prime \prime} \Phi_{1}^{\prime \prime}=\Phi_{1}^{i v} \Phi_{1}=\left(\frac{\rho A_{2} \omega_{1}^{2}}{E I_{2}} \Phi_{1}\right) \Phi_{1} \quad \Phi_{2}^{\prime \prime} \Phi_{2}^{\prime \prime}=\Phi_{2}^{i v} \Phi_{2}=\left(\frac{\rho A_{2} \omega_{2}^{2}}{E I_{2}} \Phi_{2}\right) \Phi_{2}
\end{aligned}
$$

Substituting Eq. (6) into the Lagrange's equations $[2,14]$ results:

$$
\begin{aligned}
& \left(I_{T}+\eta_{1} q_{1}^{2}+\eta_{2} q_{2}^{2}+2 \eta_{6} \cos \theta_{2}-2 \eta_{7} q_{1} \sin \theta_{2}-2 \eta_{8} q_{2} \sin \theta_{2}\right) \ddot{\theta}_{1}+\left(\eta_{5}+2 \eta_{1} q_{1}^{2}+2 \eta_{2} q_{2}^{2}+\eta_{6} \cos \theta_{2}\right. \\
& \left.-\eta_{7} q_{1} \sin \theta_{2}-\eta_{8} q_{2} \sin \theta_{2}\right) \ddot{\theta}_{2}+\left(\eta_{3}+\eta_{7} \cos \theta_{2}\right) \ddot{q}_{1}+\left(\eta_{4}+\eta_{8} \cos \theta_{2}\right) \ddot{q}_{2}-\left(2 \eta_{6} \sin \theta_{2}+2 \eta_{7} q_{1} \cos \theta_{2}\right. \\
& \left.+2 \eta_{8} q_{2} \cos \theta_{2}\right) \dot{\theta}_{1} \dot{\theta}_{2}+\left(2 \eta_{1} q_{1}-2 \eta_{7} \sin \theta_{2}\right) \dot{\theta}_{1} \dot{q}_{1}+\left(2 \eta_{2} q_{2}-2 \eta_{8} \sin \theta_{2}\right) \dot{\theta}_{1} \dot{q}_{2}+\left(4 \eta_{1} q_{1}-2 \eta_{7} \sin \theta_{2}\right) \\
& \dot{\theta}_{2} \dot{q}_{1}+\left(4 \eta_{2} q_{2}-2 \eta_{8} \sin \theta_{2}\right) \dot{\theta}_{2} \dot{q}_{2}-\left(\eta_{6} \sin \theta_{2}+\eta_{7} q_{1} \cos \theta_{2}+\eta_{8} q_{2} \cos \theta_{2}\right) \dot{\theta}_{2}^{2}=Q_{\theta_{1}} \\
& \left(\eta_{5}+2 \eta_{1} q_{1}^{2}+2 \eta_{2} q_{2}^{2}\right) \ddot{\theta}_{2}+\left(\eta_{5}+2 \eta_{1} q_{1}^{2}+2 \eta_{2} q_{2}^{2}+\eta_{6} \cos \theta_{2}-\eta_{7} q_{1} \sin \theta_{2}-\eta_{8} q_{2} \sin \theta_{2}\right) \ddot{\theta}_{1}+\left(\eta_{3}\right) \ddot{q}_{1} \\
& +\left(\eta_{4}\right) \ddot{q}_{2}+\left(4 \eta_{1} q_{1}\right) \dot{\theta}_{1} \dot{q}_{1}+\left(4 \eta_{2} q_{2}\right) \dot{\theta}_{1} \dot{q}_{2}+\left(4 \eta_{1} q_{1}\right) \dot{\theta}_{2} \dot{q}_{1}+\left(4 \eta_{2} q_{2}\right) \dot{\theta}_{2} \dot{q}_{2}+\left(\eta_{6} \sin \theta_{2}+\eta_{7} q_{1} \cos \theta_{2}\right. \\
& \left.+\eta_{8} q_{2} \cos \theta_{2}\right) \dot{\theta}_{1}^{2}=Q_{\theta_{2}} \\
& \left(2 \eta_{1}\right) \ddot{q}_{1}+\left(\eta_{3}+\eta_{7} \cos \theta_{2}\right) \ddot{\theta}_{1}+\left(\eta_{3}\right) \ddot{\theta}_{2}+\left(2 \eta_{9} \omega_{1}^{2}\right) q_{1}-\left(4 \eta_{1} q_{1}\right) \dot{\theta}_{1} \dot{\theta}_{2}-\left(\eta_{1} q_{1}+\eta_{7} \sin \theta_{2}\right) \dot{\theta}_{1}^{2} \\
& -\left(2 \eta_{1} q_{1}\right) \dot{\theta}_{2}^{2}=Q_{q_{1}} \\
& \left(2 \eta_{2}\right) \ddot{q}_{2}+\left(\eta_{4}+\eta_{8} \cos \theta_{2}\right) \ddot{\theta}_{1}+\left(\eta_{4}\right) \ddot{\theta}_{2}+\left(2 \eta_{10} \omega_{2}^{2}\right) q_{2}+\left(4 \eta_{2} q_{2}\right) \dot{\theta}_{1} \dot{\theta}_{2}+\left(\eta_{2} q_{2}+\eta_{8} \sin \theta_{2}\right) \dot{\theta}_{1}^{2} \\
& +\left(2 \eta_{2} q_{2}\right) \dot{\theta}_{2}^{2}=Q_{q_{2}}
\end{aligned}
$$


Equation (7) are the governing equations of motion for the system depicted in Fig. 1. The quantities $Q_{\theta_{1}}$ and $Q_{\theta_{2}}$ are generalized torques and the quantities $Q_{q_{1}}$ and $Q_{q_{2}}$ are generalized forces.

These equations in matrix form are written as:

$$
\begin{gathered}
{\left[\begin{array}{cccc}
m_{1} & m_{5} & m_{6} & m_{7} \\
m_{5} & m_{2} & m_{8} & m_{9} \\
m_{6} & m_{8} & m_{3} & 0 \\
m_{7} & m_{9} & 0 & m_{4}
\end{array}\right]\left\{\begin{array}{c}
\ddot{\theta}_{1} \\
\ddot{\theta}_{2} \\
\ddot{q}_{1} \\
\ddot{q}_{2}
\end{array}\right\}+\left[\begin{array}{cccc}
0 & 0 & 0 & 0 \\
0 & 0 & 0 & 0 \\
0 & 0 & 2 \eta_{9} \omega_{1}^{2} & 0 \\
0 & 0 & 0 & 2 \eta_{10} \omega_{2}^{2}
\end{array}\right]\left\{\begin{array}{c}
\theta_{1} \\
\theta_{2} \\
q_{1} \\
q_{2}
\end{array}\right\}+} \\
\left\{\begin{array}{c}
-2 n_{1} \dot{\theta}_{1} \dot{\theta}_{2}+n_{2} \dot{\theta}_{1} \dot{q}_{1}+n_{3} \dot{\theta}_{1} \dot{q}_{2}+n_{4} \dot{\theta}_{2} \dot{q}_{1}+n_{5} \dot{\theta}_{2} \dot{q}_{2}-n_{1} \dot{\theta}_{2}^{2} \\
2 n_{8} \dot{\theta}_{1} \dot{q}_{1}+2 \dot{\theta}_{9} \dot{\theta}_{2}+2 n_{8} \dot{\theta}_{2} \dot{q}_{1}+2 n_{9} \dot{\theta}_{2} \dot{q}_{2}+n_{1} \dot{\theta}_{1}^{2} \\
-2 n_{8} \dot{\theta}_{1} \dot{\theta}_{2}-n_{6} \dot{\theta}_{1}^{2}-n_{8} \dot{\theta}_{2}^{2} \\
2 n_{9} \dot{\theta}_{1} \dot{\theta}_{2}+n_{7} \dot{\theta}_{1}^{2}+n_{9} \dot{\theta}_{2}^{2}
\end{array}\right\}=\left\{\begin{array}{c}
Q_{\theta_{1}} \\
Q_{\theta_{2}} \\
Q_{q_{1}} \\
Q_{q_{2}}
\end{array}\right\}
\end{gathered}
$$

where:

$$
\begin{aligned}
& m_{1}=I_{T}+\eta_{1} q_{1}^{2}+\eta_{2} q_{2}^{2}+2 \eta_{6} \cos \theta_{2}-2 \eta_{7} q_{1} \sin \theta_{2}-2 \eta_{8} q_{2} \sin \theta_{2} \quad m_{2}=\eta_{5}+2 \eta_{1} q_{1}^{2}+2 \eta_{2} q_{2}^{2} \\
& m_{3}=2 \eta_{1} \quad m_{4}=2 \eta_{2} \quad m_{5}=\eta_{5}+2 \eta_{1} q_{1}^{2}+2 \eta_{2} q_{2}^{2}+\eta_{6} \cos \theta_{2}-\eta_{7} q_{1} \sin \theta_{2}-\eta_{8} q_{2} \sin \theta_{2} \\
& m_{6}=\eta_{3}+\eta_{7} \cos \theta_{2} \quad m_{7}=\eta_{4}+\eta_{8} \cos \theta_{2} \quad m_{8}=\eta_{3} \quad m_{9}=\eta_{4} \\
& n_{1}=\eta_{6} \sin \theta_{2}+\eta_{7} q_{1} \cos \theta_{2}+\eta_{8} q_{2} \cos \theta_{2} \quad n_{2}=2 \eta_{1} q_{1}-2 \eta_{7} \sin \theta_{2}, n_{3}=2 \eta_{2} q_{2}-2 \eta_{8} \sin \theta_{2} \\
& n_{4}=4 \eta_{1} q_{1}-2 \eta_{7} \sin \theta_{2} \quad n_{5}=4 \eta_{2} q_{2}-2 \eta_{8} \sin \theta_{2} \quad n_{6}=\eta_{1} q_{1}+\eta_{7} \sin \theta_{2} \quad n_{7}=\eta_{2} q_{2}+\eta_{8} \sin \theta_{2} \\
& n_{8}=2 \eta_{1} q_{1} \quad n_{9}=2 \eta_{2} q_{2}
\end{aligned}
$$

Let the mass matrix, $M$, be given by:

$$
M=\left[\begin{array}{cccc}
m_{1} & m_{5} & m_{6} & m_{7} \\
m_{5} & m_{2} & m_{8} & m_{9} \\
m_{6} & m_{8} & m_{3} & 0 \\
m_{7} & m_{9} & 0 & m_{4}
\end{array}\right]
$$

The inverse of $M$ is given by:

$$
M^{-1}=\left[\begin{array}{cccc}
z_{1} & z_{5} & z_{6} & z_{7} \\
z_{5} & z_{2} & z_{8} & z_{9} \\
z_{6} & z_{8} & z_{3} & z_{10} \\
z_{7} & z_{9} & z_{10} & z_{4}
\end{array}\right]
$$

where:

$$
\begin{aligned}
z_{1} & =\frac{m_{2} m_{3} m_{4}-m_{4} m_{8}^{2}-m_{3} m_{9}^{2}}{\operatorname{den} Z} \quad z_{2}=\frac{m_{1} m_{3} m_{4}-m_{4} m_{6}^{2}-m_{3} m_{7}^{2}}{\operatorname{den} Z} \\
z_{3} & =\frac{m_{1} m_{2} m_{4}-m_{4} m_{5}^{2}-m_{2} m_{7}^{2}+2 m_{5} m_{7} m_{9}-m_{1} m_{9}^{2}}{\operatorname{den} Z} \\
z_{4} & =\frac{m_{1} m_{2} m_{3}-m_{3} m_{5}^{2}-m_{2} m_{6}^{2}+2 m_{5} m_{6} m_{8}-m_{1} m_{8}^{2}}{\operatorname{den} Z} \\
z_{5} & =\frac{-m_{3} m_{4} m_{5}+m_{4} m_{6} m_{8}+m_{3} m_{7} m_{9}}{\operatorname{den} Z} \\
z_{6} & =\frac{-m_{2} m_{4} m_{6}+m_{4} m_{5} m_{8}-m_{7} m_{8} m_{9}+m_{6} m_{9}^{2}}{\operatorname{den} Z}
\end{aligned}
$$




$$
\begin{aligned}
z_{7}= & \frac{-m_{2} m_{3} m_{7}+m_{7} m_{8}^{2}+m_{3} m_{5} m_{9}-m_{6} m_{8} m_{9}}{\operatorname{den} Z} \\
z_{8}= & \frac{m_{4} m_{5} m_{6}-m_{1} m_{4} m_{8}+m_{7}^{2} m_{8}-m_{6} m_{7} m_{9}}{\operatorname{den} Z} \\
z_{9}= & \frac{m_{3} m_{5} m_{7}-m_{6} m_{7} m_{8}-m_{1} m_{3} m_{9}+m_{6}^{2} m_{9}}{\operatorname{den} Z} \\
z_{10}= & \frac{m_{2} m_{6} m_{7}-m_{5} m_{7} m_{8}-m_{5} m_{6} m_{9}+m_{1} m_{8} m_{9}}{\operatorname{den} Z} \\
\operatorname{den} Z= & m_{1} m_{2} m_{3} m_{4}-m_{3} m_{4} m_{5}^{2}-m_{2} m_{4} m_{6}^{2}-m_{2} m_{3} m_{7}^{2}+2 m_{4} m_{5} m_{6} m_{8}-m_{1} m_{4} m_{8}^{2}+m_{7}^{2} m_{8}^{2} \\
& \quad+2 m_{3} m_{5} m_{7} m_{9}-2 m_{6} m_{7} m_{8} m_{9}-m_{1} m_{3} m_{9}^{2}+m_{6}^{2} m_{9}^{2}
\end{aligned}
$$

Multiplying Eq. (8) to the left by $M^{-1}$ and rearranging results:

$$
\begin{aligned}
& \ddot{\theta}_{1}+2 z_{6} \eta_{9} \omega_{1}^{2} q_{1}+2 z_{7} \eta_{10} \omega_{2}^{2} q_{2}+z_{1}\left(-2 n_{1} \dot{\theta}_{1} \dot{\theta}_{2}+n_{2} \dot{\theta}_{1} \dot{q}_{1}+n_{3} \dot{\theta}_{1} \dot{q}_{2}+n_{4} \dot{\theta}_{2} \dot{q}_{1}+n_{5} \dot{\theta}_{2} \dot{q}_{2}-n_{1} \dot{\theta}_{2}^{2}\right) \\
& +z_{5}\left(2 n_{8} \dot{\theta}_{1} \dot{q}_{1}+2 n_{9} \dot{\theta}_{1} \dot{q}_{2}+2 n_{8} \dot{\theta}_{2} \dot{q}_{1}+2 n_{9} \dot{\theta}_{2} \dot{q}_{2}+n_{1} \dot{\theta}_{1}^{2}\right)-z_{6}\left(2 n_{8} \dot{\theta}_{1} \dot{\theta}_{2}+n_{6} \dot{\theta}_{1}^{2}+n_{8} \dot{\theta}_{2}^{2}\right) \\
& +z_{7}\left(2 n_{9} \dot{\theta}_{1} \dot{\theta}_{2}+n_{7} \dot{\theta}_{1}^{2}+n_{9} \dot{\theta}_{2}^{2}\right)=z_{1} Q_{\theta_{1}}+z_{5} Q_{\theta_{2}}+z_{6} Q_{q_{1}}+z_{7} Q_{q_{2}} \\
& \ddot{\theta}_{2}+2 z_{8} \eta_{9} \omega_{1}^{2} q_{1}+2 z_{9} \eta_{10} \omega_{2}^{2} q_{2}+z_{5}\left(-2 n_{1} \dot{\theta}_{1} \dot{\theta}_{2}+n_{2} \dot{\theta}_{1} \dot{q}_{1}+n_{3} \dot{\theta}_{1} \dot{q}_{2}+n_{4} \dot{\theta}_{2} \dot{q}_{1}+n_{5} \dot{\theta}_{2} \dot{q}_{2}-n_{1} \dot{\theta}_{2}^{2}\right) \\
& +z_{2}\left(2 n_{8} \dot{\theta}_{1} \dot{q}_{1}+2 n_{9} \dot{\theta}_{1} \dot{q}_{2}+2 n_{8} \dot{\theta}_{2} \dot{q}_{1}+2 n_{9} \dot{\theta}_{2} \dot{q}_{2}+n_{1} \dot{\theta}_{1}^{2}\right)-z_{8}\left(2 n_{8} \dot{\theta}_{1} \dot{\theta}_{2}+n_{6} \dot{\theta}_{1}^{2}+n_{8} \dot{\theta}_{2}^{2}\right) \\
& +z_{9}\left(2 n_{9} \dot{\theta}_{1} \dot{\theta}_{2}+n_{7} \dot{\theta}_{1}^{2}+n_{9} \dot{\theta}_{2}^{2}\right)=z_{5} Q_{\theta_{1}}+z_{2} Q_{\theta_{2}}+z_{8} Q_{q_{1}}+z_{9} Q_{q_{2}} \\
& \ddot{q}_{1}+2 z_{3} \eta_{9} \omega_{1}^{2} q_{1}+2 z_{10} \eta_{10} \omega_{2}^{2} q_{2}+z_{6}\left(-2 n_{1} \dot{\theta}_{1} \dot{\theta}_{2}+n_{2} \dot{\theta}_{1} \dot{q}_{1}+n_{3} \dot{\theta}_{1} \dot{q}_{2}+n_{4} \dot{\theta}_{2} \dot{q}_{1}+n_{5} \dot{\theta}_{2} \dot{q}_{2}-n_{1} \dot{\theta}_{2}^{2}\right) \\
& +z_{8}\left(2 n_{8} \dot{\theta}_{1} \dot{q}_{1}+2 n_{9} \dot{\theta}_{1} \dot{q}_{2}+2 n_{8} \dot{\theta}_{2} \dot{q}_{1}+2 n_{9} \dot{\theta}_{2} \dot{q}_{2}+n_{1} \dot{\theta}_{1}^{2}\right)-z_{3}\left(2 n_{8} \dot{\theta}_{1} \dot{\theta}_{2}+n_{6} \dot{\theta}_{1}^{2}+n_{8} \dot{\theta}_{2}^{2}\right) \\
& +z_{10}\left(2 n_{9} \dot{\theta}_{1} \dot{\theta}_{2}+n_{7} \dot{\theta}_{1}^{2}+n_{9} \dot{\theta}_{2}^{2}\right)=z_{6} Q_{\theta_{1}}+z_{8} Q_{\theta_{2}}+z_{3} Q_{q_{1}}+z_{10} Q_{q_{2}} \\
& \ddot{q}_{2}+2 z_{10} \eta_{9} \omega_{1}^{2} q_{1}+2 z_{4} \eta_{10} \omega_{2}^{2} q_{2}+z_{7}\left(-2 n_{1} \dot{\theta}_{1} \dot{\theta}_{2}+n_{2} \dot{\theta}_{1} \dot{q}_{1}+n_{3} \dot{\theta}_{1} \dot{q}_{2}+n_{4} \dot{\theta}_{2} \dot{q}_{1}+n_{5} \dot{\theta}_{2} \dot{q}_{2}-n_{1} \dot{\theta}_{2}^{2}\right) \\
& +z_{9}\left(2 n_{8} \dot{\theta}_{1} \dot{q}_{1}+2 n_{9} \dot{\theta}_{1} \dot{q}_{2}+2 n_{8} \dot{\theta}_{2} \dot{q}_{1}+2 n_{9} \dot{\theta}_{2} \dot{q}_{2}+n_{1} \dot{\theta}_{1}^{2}\right)-z_{10}\left(2 n_{8} \dot{\theta}_{1} \dot{\theta}_{2}+n_{6} \dot{\theta}_{1}^{2}+n_{8} \dot{\theta}_{2}^{2}\right) \\
& +z_{4}\left(2 n_{9} \dot{\theta}_{1} \dot{\theta}_{2}+n_{7} \dot{\theta}_{1}^{2}+n_{9} \dot{\theta}_{2}^{2}\right)=z_{7} Q_{\theta_{1}}+z_{9} Q_{\theta_{2}}+z_{10} Q_{q_{1}}+z_{4} Q_{q_{2}}
\end{aligned}
$$

Equation (9) are decoupled in the acelerations and ready to be written in state space form to be numerically integrated. In state space form, Eq. (9) can be writen as:

$$
\begin{aligned}
\dot{x}_{1}= & x_{2} \\
\dot{x}_{2}= & z_{1 x} Q_{\theta_{1}}+z_{5 x} Q_{\theta_{2}}+z_{6 x} Q_{q_{1}}+z_{7 x} Q_{q_{2}}-2 z_{6 x} \eta_{9} \omega_{1}^{2} x_{5}+2 z_{7 x} \eta_{10} \omega_{2}^{2} x_{7}-z_{1 x}\left(-2 n_{1 x} x_{2} x_{4}+n_{2 x} x_{2} x_{6}\right. \\
& \left.+n_{3 x} x_{2} x_{8}+n_{4 x} x_{4} x_{6}+n_{5 x} x_{4} x_{8}-n_{1 x} x_{4}^{2}\right)-z_{5 x}\left(2 n_{8 x} x_{2} x_{6}+2 n_{9 x} x_{2} x_{8}+2 n_{8 x} x_{4} x_{6}\right. \\
& \left.+2 n_{9 x} x_{4} x_{8}+n_{x 1} x_{2}^{2}\right)+z_{6 x}\left(2 n_{8 x} x_{2} x_{4}+n_{6 x} x_{2}^{2}+n_{8 x} x_{4}^{2}\right)-z_{7 x}\left(2 n_{9 x} x_{2} x_{4}+n_{7 x} x_{2}^{2}+n_{9 x} x_{4}^{2}\right) \\
\dot{x}_{3}= & x_{4} \\
\dot{x}_{4}= & z_{5 x} Q_{\theta_{1}}+z_{2 x} Q_{\theta_{2}}+z_{8 x} Q_{q_{1}}+z_{9 x} Q_{q_{2}}-2 z_{8 x} \eta_{9} \omega_{1}^{2} x_{5}+2 z_{9 x} \eta_{10} \omega_{2}^{2} x_{7}-z_{5 x}\left(-2 n_{1 x} x_{2} x_{4}+n_{2 x} x_{2} x_{6}\right. \\
& \left.+n_{3 x} x_{2} x_{8}+n_{4 x} x_{4} x_{6}+n_{5 x} x_{4} x_{8}-n_{1 x} x_{4}^{2}\right)-z_{2 x}\left(2 n_{8 x} x_{2} x_{6}+2 n_{9 x} x_{2} x_{8}+2 n_{8 x} x_{4} x_{6}\right. \\
& \left.+2 n_{9 x} x_{4} x_{8}+n_{x 1} x_{2}^{2}\right)+z_{8 x}\left(2 n_{8 x} x_{2} x_{4}+n_{6 x} x_{2}^{2}+n_{8 x} x_{4}^{2}\right)-z_{9 x}\left(2 n_{9 x} x_{2} x_{4}+n_{7 x} x_{2}^{2}+n_{9 x} x_{4}^{2}\right) \\
\dot{x}_{5}= & x_{6} \\
\dot{x}_{6}= & z_{6 x} Q_{\theta_{1}}+z_{8 x} Q_{\theta_{2}}+z_{3 x} Q_{q_{1}}+z_{10 x} Q_{q_{2}}-2 z_{3 x} \eta_{9} \omega_{1}^{2} x_{5}+2 z_{10 x} \eta_{10} \omega_{2}^{2} x_{7}-z_{6 x}\left(-2 n_{1 x} x_{2} x_{4}+n_{2 x} x_{2} x_{6}\right.
\end{aligned}
$$




$$
\begin{aligned}
& \left.+n_{3 x} x_{2} x_{8}+n_{4 x} x_{4} x_{6}+n_{5 x} x_{4} x_{8}-n_{1 x} x_{4}^{2}\right)-z_{8 x}\left(2 n_{8 x} x_{2} x_{6}+2 n_{9 x} x_{2} x_{8}+2 n_{8 x} x_{4} x_{6}\right. \\
& \left.+2 n_{9 x} x_{4} x_{8}+n_{x 1} x_{2}^{2}\right)+z_{3 x}\left(2 n_{8 x} x_{2} x_{4}+n_{6 x} x_{2}^{2}+n_{8 x} x_{4}^{2}\right)-z_{10 x}\left(2 n_{9 x} x_{2} x_{4}+n_{7 x} x_{2}^{2}+n_{9 x} x_{4}^{2}\right) \\
\dot{x}_{7}= & x_{8} \\
\dot{x}_{8}= & z_{7 x} Q_{\theta_{1}}+z_{9 x} Q_{\theta_{2}}+z_{10 x} Q_{q_{1}}+z_{4 x} Q_{q_{2}}-2 z_{10 x} \eta_{9} \omega_{1}^{2} x_{5}+2 z_{4 x} \eta_{10} \omega_{2}^{2} x_{7}-z_{7 x}\left(-2 n_{1 x} x_{2} x_{4}+n_{2 x} x_{2} x_{6}\right. \\
& \left.+n_{3 x} x_{2} x_{8}+n_{4 x} x_{4} x_{6}+n_{5 x} x_{4} x_{8}-n_{1 x} x_{4}^{2}\right)-z_{9 x}\left(2 n_{8 x} x_{2} x_{6}+2 n_{9 x} x_{2} x_{8}+2 n_{8 x} x_{4} x_{6}\right. \\
& \left.+2 n_{9 x} x_{4} x_{8}+n_{x 1} x_{2}^{2}\right)+z_{10 x}\left(2 n_{8 x} x_{2} x_{4}+n_{6 x} x_{2}^{2}+n_{8 x} x_{4}^{2}\right)-z_{4 x}\left(2 n_{9 x} x_{2} x_{4}+n_{7 x} x_{2}^{2}+n_{9 x} x_{4}^{2}\right)
\end{aligned}
$$

In Eq. (10), the states considered are: $x_{1}=\theta_{1}, x_{2}=\dot{\theta}_{1}, x_{3}=\theta_{2}, x_{4}=\dot{\theta}_{2}, x_{5}=q_{1}, x_{6}=\dot{q}_{1}, x_{7}=q_{2}$ and $x_{8}=\dot{q}_{2}$. In this same equation, $z_{i x}$ and $n_{i x}$ are $z_{i}$ and $n_{i}$ expressed as functions of the states $x_{1}$ to $x_{8}$.

The state dependent matrices $A(x)$ and $B(x)$ used in this work are:

$$
A(x)=\left[\begin{array}{cccccccc}
0 & 1 & 0 & 0 & 0 & 0 & 0 & 0 \\
0 & A_{1} & 0 & A_{2} & A_{3} & A_{4} & A_{5} & A_{6} \\
0 & 0 & 0 & 1 & 0 & 0 & 0 & 0 \\
0 & A_{7} & 0 & A_{8} & A_{9} & A_{10} & A_{11} & A_{12} \\
0 & 0 & 0 & 0 & 0 & 1 & 0 & 0 \\
0 & A_{13} & 0 & A_{14} & A_{15} & A_{16} & A_{17} & A_{18} \\
0 & 0 & 0 & 0 & 0 & 0 & 0 & 1 \\
0 & A_{19} & 0 & A_{20} & A_{21} & A_{22} & A_{23} & A_{24}
\end{array}\right] \text { and } B(x)=\left[\begin{array}{cccc}
0 & 0 & 0 & 0 \\
z_{1 x} & z_{5 x} & z_{6 x} & z_{7 x} \\
0 & 0 & 0 & 0 \\
z_{5 x} & z_{2 x} & z_{8 x} & z_{9 x} \\
0 & 0 & 0 & 0 \\
z_{6 x} & z_{8 x} & z_{3 x} & z_{10 x} \\
0 & 0 & 0 & 0 \\
z_{7 x} & z_{9 x} & z_{10 x} & z_{4 x}
\end{array}\right]
$$

where:

$$
\begin{aligned}
& A_{1}=\left(z_{6 x} n_{6 x}-z_{5 x} n_{1 x}-z_{7 x} n_{7 x}\right) x_{2}+\left(2 z_{1 x} n_{1 x}+2 z_{6 x} n_{8 x}-2 z_{7 x} n_{9 x}\right) x_{4} \\
& A_{2}=\left(z_{1 x} n_{1 x}+z_{6 x} n_{8 x}-z_{7 x} n_{9 x}\right) x_{4} \\
& A_{3}=-2 z_{6 x} \eta_{9} \omega_{1}^{2} \\
& A_{4}=\left(-z_{1 x} n_{2 x}-2 z_{5 x} n_{8 x}\right) x_{2}+\left(-2 z_{5 x} n_{8 x}-z_{1 x} n_{4 x}\right) x_{4} \\
& A_{5}=2 z_{7 x} \eta_{10} \omega_{2}^{2} \\
& A_{6}=\left(-z_{1 x} n_{3 x}-2 z_{5 x} n_{9 x}\right) x_{2}+\left(-z_{1 x} n_{5 x}-2 z_{5 x} n_{9 x}\right) x_{4} \\
& A_{7}=\left(z_{8 x} n_{6 x}-z_{2 x} n_{1 x}-z_{9 x} n_{7 x}\right) x_{2}+\left(2 z_{5 x} n_{1 x}+2 z_{8 x} n_{8 x}-2 z_{9 x} n_{9 x}\right) x_{4} \\
& A_{8}=\left(z_{5 x} n_{1 x}+z_{8 x} n_{8 x}-z_{9 x} n_{9 x}\right) x_{4} \\
& A_{9}=-2 z_{8 x} \eta_{9} \omega_{1}^{2} \\
& A_{10}=\left(-z_{5 x} n_{2 x}-2 z_{2 x} n_{8 x}\right) x_{2}+\left(-z_{5 x} n_{4 x}-2 z_{2 x} n_{8 x}\right) x_{4} \\
& A_{11}=2 z_{9 x} \eta_{10} \omega_{2}^{2} \\
& A_{12}=\left(-z_{5 x} n_{3 x}-2 z_{2 x} n_{9 x}\right) x_{2}+\left(-z_{5 x} n_{5 x}-2 z_{2 x} n_{9 x}\right) x_{4} \\
& A_{13}=\left(z_{3 x} n_{6 x}-z_{8 x} n_{1 x}-z_{10 x} n_{7 x}\right) x_{2}+\left(2 z_{6 x} n_{1 x}+2 z_{3 x} n_{8 x}-2 z_{10 x} n_{9 x}\right) x_{4} \\
& A_{14}=\left(z_{6 x} n_{1 x}+z_{3 x} n_{8 x}-z_{10 x} n_{9 x}\right) x_{4} \\
& A_{15}=-2 z_{3 x} \eta_{9} \omega_{1}^{2} \\
& A_{16}=\left(-z_{6 x} n_{2 x}-2 z_{8 x} n_{8 x}\right) x_{2}+\left(-z_{6 x} n_{4 x}-2 z_{8 x} n_{8 x}\right) x_{4} \\
& A_{17}=2 z_{10 x} \eta_{10} \omega_{2}^{2} \\
& A_{18}=\left(-z_{6 x} n_{3 x}-2 z_{8 x} n_{9 x}\right) x_{2}+\left(-z_{6 x} n_{5 x}-2 z_{8 x} n_{9 x}\right) x_{4}
\end{aligned}
$$




$$
\begin{aligned}
& A_{19}=\left(z_{10 x} n_{6 x}-z_{9 x} n_{1 x}-z_{4 x} n_{7 x}\right) x_{2}+\left(2 z_{7 x} n_{1 x}+2 z_{10 x} n_{8 x}-2 z_{4 x} n_{9 x}\right) x_{4} \\
& A_{20}=\left(z_{7 x} n_{1 x}+z_{10 x} n_{8 x}-z_{4 x} n_{9 x}\right) x_{4} \\
& A_{21}=-2 z_{10 x} \eta_{9} \omega_{1}^{2} \\
& A_{22}=\left(-z_{7 x} n_{2 x}-2 z_{9 x} n_{8 x}\right) x_{2}+\left(-z_{7 x} n_{4 x}-2 z_{9 x} n_{8 x}\right) x_{4} \\
& A_{23}=2 z_{4 x} \eta_{10} \omega_{2}^{2} \\
& A_{24}=\left(-z_{7 x} n_{3 x}-2 z_{9 x} n_{9 x}\right) x_{2}+\left(-z_{7 x} n_{5 x}-2 z_{9 x} n_{9 x}\right) x_{4}
\end{aligned}
$$

\section{The State-Dependent Riccati Equation (SDRE) control}

The State-Dependent Riccati Equation (SDRE) approach to nonlinear system control relies on representing a nonlinear system's dynamics with state-dependent coefficient matrices that can be inserted into state-dependent Riccati equations to generate a feedback law [16].

The main idea of this method is to represent the nonlinear system:

$$
\dot{x}=f(x)+B(x) u
$$

in the form:

$$
\dot{x}=A(x) x+B(x) u
$$

The feedback law is given by:

$$
u=-R^{-1}(x) B^{T}(x) P(x) x
$$

where $P(x)$ is obtained from the SDRE:

$$
P(x) A(x)+A^{T}(x) P(x)+Q(x)-P(x) B(x) R^{-1}(x) B^{T}(x) P(x)=0
$$

In Eqs (12) and (13), $Q(x)$ and $R(x)$ are design parameters that satisfy the positive definiteness condition:

$$
Q(x)>0 \quad R(x)>0
$$

Equation (10), in state space form, can be written in the form given by Eq. (11) with both $A$ and $B$ matrices state dependent.

\section{The control torques}

Using Eq. (12), the control torques are given by:

$$
\begin{aligned}
& u=-\left[\begin{array}{cccc}
\frac{1}{R_{1}} & 0 & 0 & 0 \\
0 & \frac{1}{R_{2}} & 0 & 0 \\
0 & 0 & \frac{1}{R_{3}} & 0 \\
0 & 0 & 0 & \frac{1}{R_{4}}
\end{array}\right]\left[\begin{array}{cccccccc}
0 & z_{1 x} & 0 & z_{5 x} & 0 & z_{6 x} & 0 & z_{7 x} \\
0 & z_{5 x} & 0 & z_{2 x} & 0 & z_{8 x} & 0 & z_{9 x} \\
0 & z_{6 x} & 0 & z_{8 x} & 0 & z_{3 x} & 0 & z_{10 x} \\
0 & z_{7 x} & 0 & z_{9 x} & 0 & z_{10 x} & 0 & z_{4 x}
\end{array}\right] \\
& {\left[\begin{array}{cccccccc}
P_{1} & P_{2} & P_{3} & P_{4} & P_{5} & P_{6} & P_{7} & P_{8} \\
P_{9} & P_{10} & P_{11} & P_{12} & P_{13} & P_{14} & P_{15} & P_{16} \\
P_{17} & P_{18} & P_{19} & P_{20} & P_{21} & P_{22} & P_{23} & P_{24} \\
P_{25} & P_{26} & P_{27} & P_{28} & P_{29} & P_{30} & P_{31} & P_{32} \\
P_{33} & P_{34} & P_{35} & P_{36} & P_{37} & P_{38} & P_{39} & P_{40} \\
P_{41} & P_{42} & P_{43} & P_{44} & P_{45} & P_{46} & P_{47} & P_{48} \\
P_{49} & P_{50} & P_{51} & P_{52} & P_{53} & P_{54} & P_{55} & P_{56} \\
P_{57} & P_{58} & P_{59} & P_{60} & P_{61} & P_{62} & P_{63} & P_{64}
\end{array}\right]\left\{\begin{array}{l}
x_{1} \\
x_{2} \\
x_{3} \\
x_{4} \\
x_{5} \\
x_{6} \\
x_{7} \\
x_{8}
\end{array}\right\}}
\end{aligned}
$$


or:

$$
u=-\left[\begin{array}{cccccccc}
K_{1} & K_{2} & K_{3} & K_{4} & K_{5} & K_{6} & K_{7} & K_{8} \\
K_{9} & K_{10} & K_{11} & K_{12} & K_{13} & K_{14} & K_{15} & K_{16} \\
K_{17} & K_{18} & K_{19} & K_{20} & K_{21} & K_{22} & K_{23} & K_{24} \\
K_{25} & K_{26} & K_{27} & K_{28} & K_{29} & K_{30} & K_{31} & K_{32}
\end{array}\right]\left\{\begin{array}{l}
x_{1} \\
x_{2} \\
x_{3} \\
x_{4} \\
x_{5} \\
x_{6} \\
x_{7} \\
x_{8}
\end{array}\right\}
$$

\section{State estimation using SDRE}

The basic idea of estimating the state vector, $\mathbf{x}$, from the output vector, $\mathbf{y}$, is to build a new system with essentially the same structure as the original system:

$$
\dot{x}=A(x) x+B(x) u
$$

and to drive this new system with an input which is proportional to the error between the actual plant output (known or measured quantities) and the estimated output.

In mathematical terms, let $\hat{x}$ denote the vector of state estimate and let the corresponding approximation to the estimated output be given by:

$$
\hat{y}=C \hat{x}
$$

The estimated states are generated by a system of the form:

$$
\dot{\hat{x}}=A(\hat{x}) \hat{x}+B(\hat{x}) \hat{u}+L(y-\hat{y})
$$

In Eq. (15), the term $L(y-\hat{y})$ correct the estimation equation with a feedback from the estimation error. If matrix $L$ is chosen carefully, it seems reasonable that one can make $\hat{x}$ in the last equation track $x$ closely.

Suppose the estimation error given by:

$$
e=x-\hat{x}
$$

is nonzero at some time $t$. Then, the difference:

$$
y-\hat{y}=C e
$$

is also likely to be nonzero.

For certain choices of $L$, the resulting forcing term $L(y-\hat{y})$ should drive $x$ in a direction that decreases the error given by Eq. (16). $L$ is the unknown observer gain.

The general idea is given in the Fig. 2.

The performance indicator is given by:

$$
\hat{J}=\frac{1}{2} \int_{0}^{\infty}\left(\hat{x}^{T} Q_{M} \hat{x}+\hat{u}^{T} R_{N} \hat{u}\right) d t
$$

and the optimal (suboptimal) control $\hat{u}$ given by:

$$
\hat{u}=-L(\hat{x}) \hat{x}
$$

The optimal (suboptimal) feedback gain is given by:

$$
L(\hat{x})=R_{N}^{-1} B^{T}(\hat{x}) P(\hat{x})
$$

with $P(\hat{x})$ obtained by solving the state-dependent Riccati equation:

$$
P(\hat{x}) A(\hat{x})+A^{T}(\hat{x}) P(\hat{x})-P(\hat{x}) B(\hat{x}) R_{N}^{-1} B^{T}(\hat{x}) P(\hat{x})+Q_{M}=0
$$


Table 1

Initial condition assumed in the numerical simulations

\begin{tabular}{|c|c|c|c|c|c|c|c|c|c|}
\hline \multicolumn{2}{|c|}{ Initial Conditions (IC) } & $\theta_{1}$ & $\dot{\theta}_{1}$ & $\theta_{2}$ & $\dot{\theta}_{2}$ & $q_{1}$ & $\dot{q}_{1}$ & $q_{2}$ & $\dot{q}_{2}$ \\
\hline \multirow[t]{2}{*}{ Same IC } & "real" & $200^{\circ}$ & $0 \mathrm{rad} / \mathrm{s}$ & $50^{\circ}$ & $0 \mathrm{rad} / \mathrm{s}$ & $0 \mathrm{~m}$ & $0 \mathrm{~m} / \mathrm{s}$ & $0 \mathrm{~m}$ & $0 \mathrm{~m} / \mathrm{s}$ \\
\hline & estimated & $200^{\circ}$ & $0 \mathrm{rad} / \mathrm{s}$ & $50^{\circ}$ & $0 \mathrm{rad} / \mathrm{s}$ & $0 \mathrm{~m}$ & $0 \mathrm{~m} / \mathrm{s}$ & - & - \\
\hline \multirow[t]{2}{*}{ Different IC } & "real" & $200^{\circ}$ & $0 \mathrm{rad} / \mathrm{s}$ & $50^{\circ}$ & $0 \mathrm{rad} / \mathrm{s}$ & $0 \mathrm{~m}$ & $0 \mathrm{~m} / \mathrm{s}$ & $0 \mathrm{~m}$ & $0 \mathrm{~m} / \mathrm{s}$ \\
\hline & estimated & $0^{\circ}$ & $0 \mathrm{rad} / \mathrm{s}$ & $0^{\circ}$ & $0 \mathrm{rad} / \mathrm{s}$ & $0 \mathrm{~m}$ & $0 \mathrm{~m} / \mathrm{s}$ & - & - \\
\hline
\end{tabular}

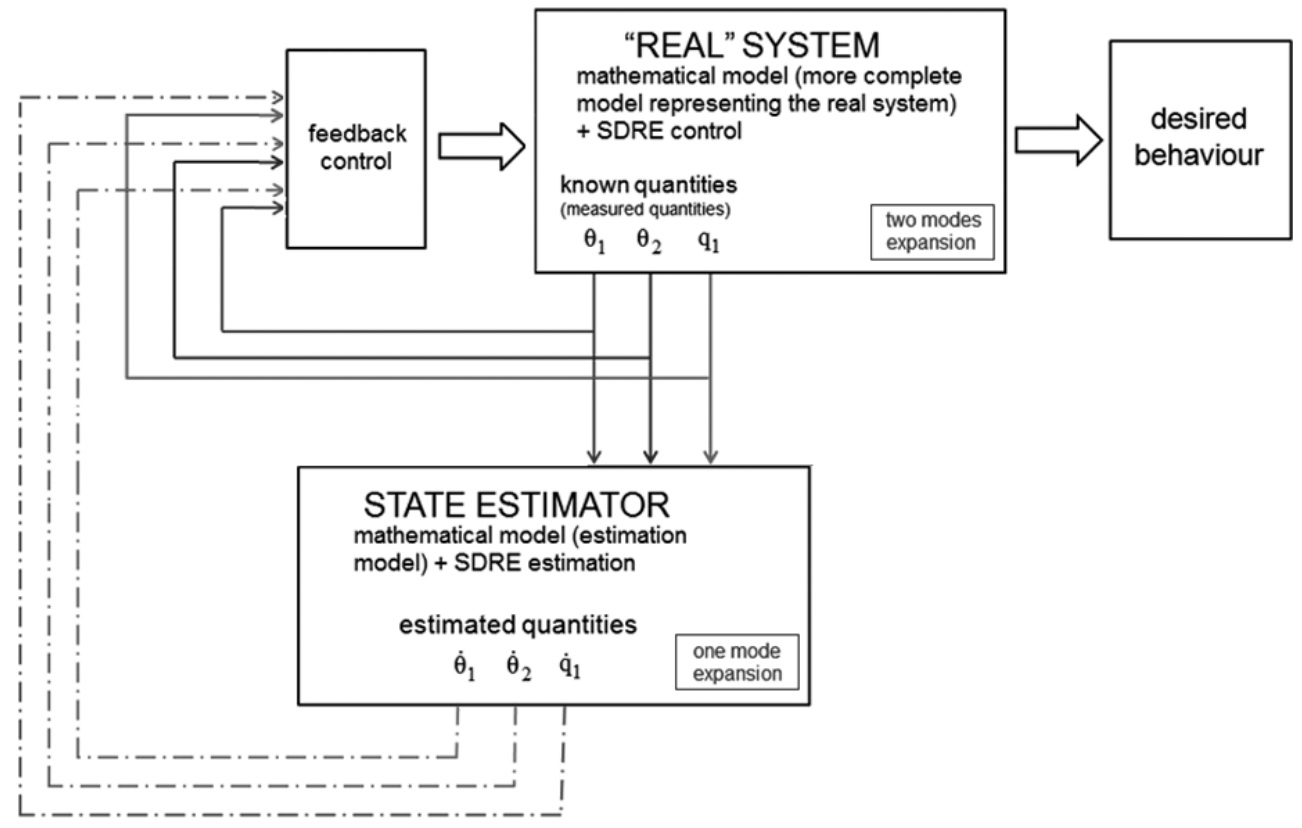

Fig. 2. The general idea for nonlinear control and nonlinear estimation using SDRE.

\section{Numerical results}

The numerical integrator used in this work is the fourth order Runge-Kutta with time step of $0.001 \mathrm{~s}$. Since matrices $A(x)$ and $B(x)$ are state dependant, it is necessary to solve the Riccati equation each time step (the suboptimal gains are time varying). This procedure makes calculations very slow for large simulation times.

In the numerical simulations different initial values are considered for the angles $\theta_{1}$ and $\theta_{2}$ and the SDRE control law is used to bring both variables to zero. This deviation from zero is considered as a position error that must be corrected. At the same time that the controller is correcting the angles it is also used to eliminate the vibration on the flexible link. The different initial conditions chosen for $\theta_{1}$ and $\theta_{2}$ intend to simulate the system in conditions into which the influence of the nonlinear terms in Eqs (9) or (10) is important.

The cases considered are presented in Table 1.

In this table and in the figures, "real" represents the mathematical model that emulates the real system and considers two modes expansion for the beam deflection and estimated represents the mathematical model with only one mode expansion. The one mode expansion model is used to estimate the velocities in the more complete two modes expansion model. All the gains used for estimation and control are calculated using the one mode mathematical model for $A(x)$ and $B(x)$ since in real systems this data is unknown.

The one mode expansion mathematical model used for estimation can be obtained from Eq. (9) by eliminating Eq. (9d) and all the references to variable $q_{2}$ and its time derivative in Eqs (9a) to (9c).

Table 2 presents the parameters values used in the numerical simulations. 
Table 2

Parameters values used in the numerical simulations

\begin{tabular}{lcl}
\hline & Parameters & Value \\
\hline Aluminum beams & $\mathrm{E}$ & $0.710^{11} \mathrm{~N} / \mathrm{m}^{2}$ \\
\multirow{3}{*}{ Rigid beam } & $\rho$ & $2700 \mathrm{Kg} / \mathrm{m}^{3}$ \\
& Height (beam cross section) & $0.0300 \mathrm{~m}$ \\
& Width (beam cross section) & $0.0300 \mathrm{~m}$ \\
Flexible beam & $L_{1}$ & $0.3000 \mathrm{~m}$ \\
& Height (beam cross section) & $0.0300 \mathrm{~m}$ \\
& Width (beam cross section) & $0.0015 \mathrm{~m}$ \\
& $L_{2}$ & $2.0000 \mathrm{~m}$ \\
\hline
\end{tabular}

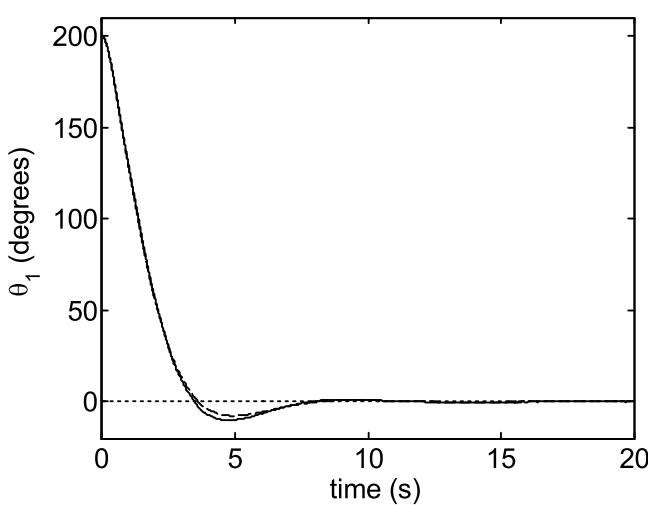

Fig. 3. Estimated (- - ) and "real" (-) behavior of measured variable $\theta_{1}$ considering same initial conditions.

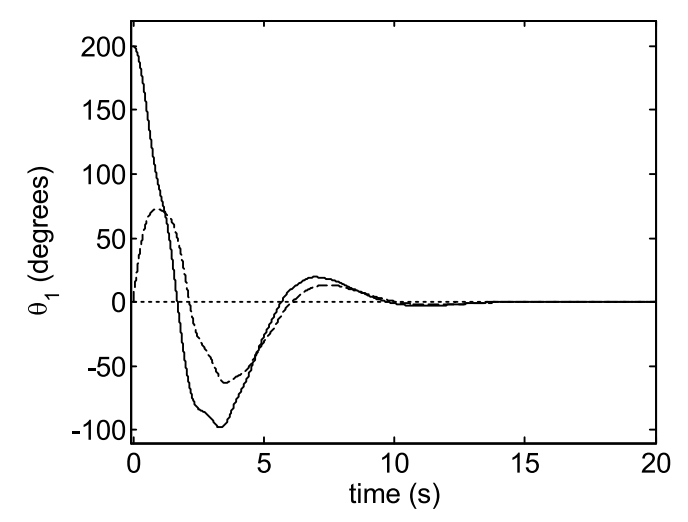

Fig. 4. Estimated (- - -) and "real" (-) behavior of measured variable $\theta_{1}$ considering different initial conditions.

The $R_{N}$ and $Q_{M}$ estimator matrices used in the numerical simulations are chosen by trial and error and given by:

$$
R_{N}=\left[\begin{array}{lll}
3 & 0 & 0 \\
0 & 3 & 0 \\
0 & 0 & 2
\end{array}\right] \quad Q_{M}=\left[\begin{array}{cccccc}
2.5 & 0 & 0 & 0 & 0 & 0 \\
0 & 1 & 0 & 0 & 0 & 0 \\
0 & 0 & 5 & 0 & 0 & 0 \\
0 & 0 & 0 & 1 & 0 & 0 \\
0 & 0 & 0 & 0 & 2000 & 0 \\
0 & 0 & 0 & 0 & 0 & 2
\end{array}\right]
$$

These matrices were used for all the numerical simulations. The same gains obtained using these matrices are also used in the SDRE control. The variable $q_{2}$ and its time derivative are neither measured nor estimated and therefore not controlled.

The measurement matrix $C$ is given by:

$$
C=\left[\begin{array}{llllll}
1 & 0 & 0 & 0 & 0 & 0 \\
0 & 0 & 0 & 0 & 0 & 0 \\
0 & 0 & 1 & 0 & 0 & 0 \\
0 & 0 & 0 & 0 & 0 & 0 \\
0 & 0 & 0 & 0 & 1 & 0 \\
0 & 0 & 0 & 0 & 0 & 0
\end{array}\right]
$$

where only the positions are measured (and not the velocities).

Using this matrix $C$, the estimated output equation is:

$$
\hat{y}=C \hat{x}=\left[\begin{array}{cccccc}
1 & 0 & 0 & 0 & 0 & 0 \\
0 & 0 & 0 & 0 & 0 & 0 \\
0 & 0 & 1 & 0 & 0 & 0 \\
0 & 0 & 0 & 0 & 0 & 0 \\
0 & 0 & 0 & 0 & 1 & 0 \\
0 & 0 & 0 & 0 & 0 & 0
\end{array}\right]\left\{\begin{array}{l}
\hat{x}_{1} \\
\hat{x}_{2} \\
\hat{x}_{3} \\
\hat{x}_{4} \\
\hat{x}_{5} \\
\hat{x}_{6}
\end{array}\right\}=\left\{\begin{array}{c}
\hat{x}_{1} \\
0 \\
\hat{x}_{3} \\
0 \\
\hat{x}_{5} \\
0
\end{array}\right\}
$$




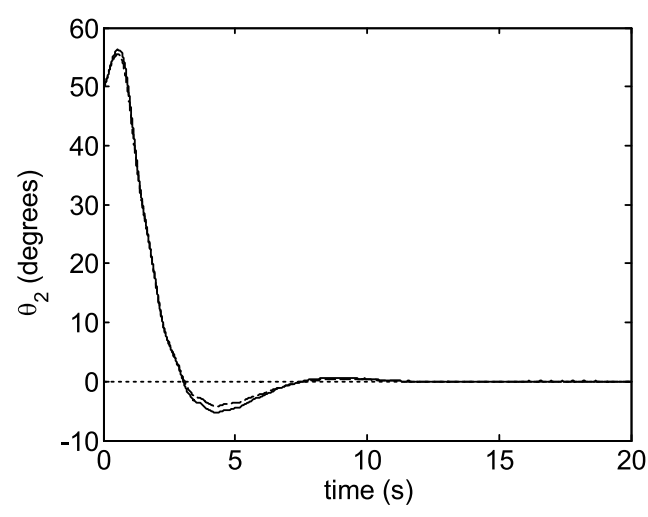

Fig. 5. Estimated (- - ) and "real" (-) behavior of measured variable $\theta_{2}$ considering same initial conditions.

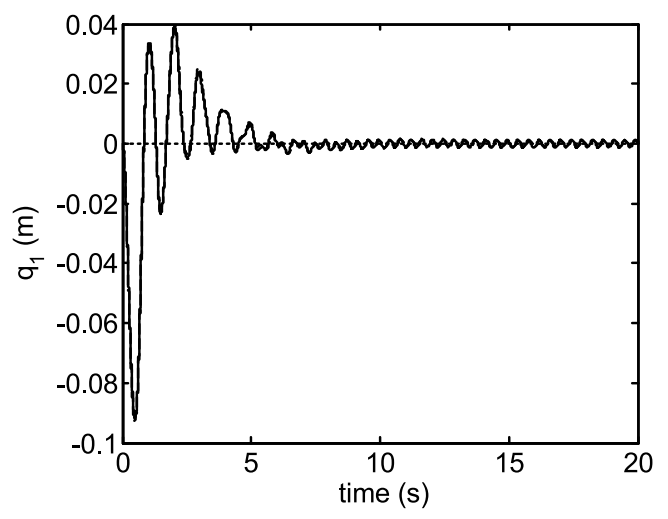

Fig. 7. Estimated (- - -) and "real" (-) behavior of measured variable $q_{1}$ considering same initial conditions.

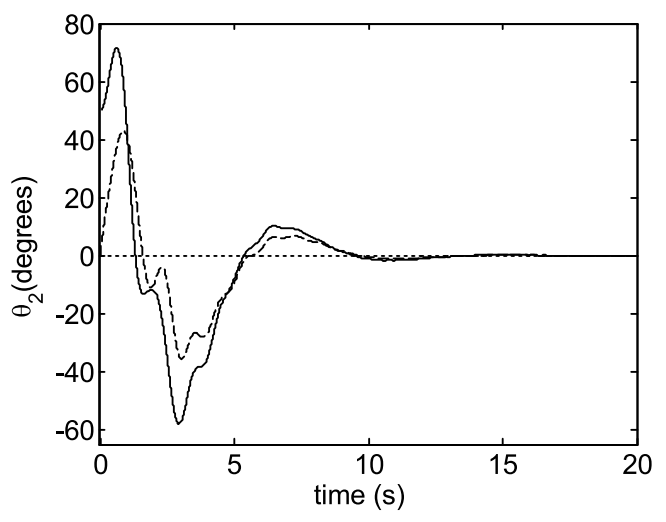

Fig. 6. Estimated (- - ) and "real" (-) behavior of measured variable $\theta_{2}$ considering same initial conditions.

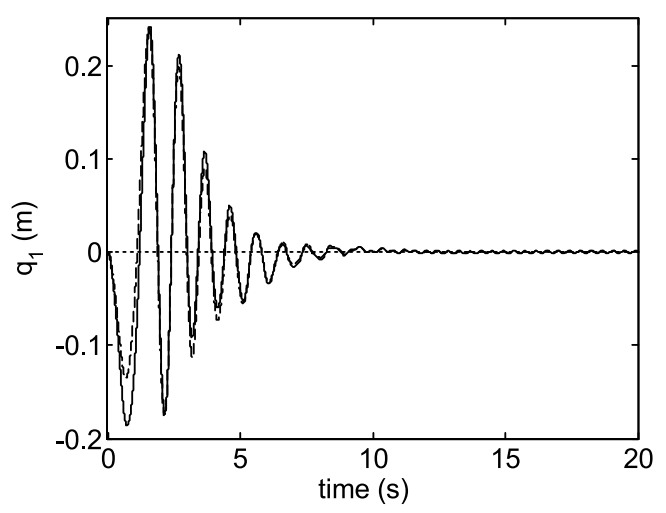

Fig. 8. Estimated (- - -) and "real" (-) behavior of measured variable $q_{1}$ considering different initial conditions.

and the terms to be included in the estimation equations are:

$$
L(\hat{x})(y-\hat{y})=\left[\begin{array}{cccccc}
L_{1} & L_{2} & L_{3} & L_{4} & L_{5} & L_{6} \\
L_{7} & L_{8} & L_{9} & L_{10} & L_{11} & L_{12} \\
L_{13} & L_{14} & L_{15} & L_{16} & L_{17} & L_{18}
\end{array}\right]\left\{\begin{array}{c}
x_{1}-\hat{x}_{1} \\
0 \\
x_{3}-\hat{x}_{3} \\
0 \\
x_{5}-\hat{x}_{5} \\
0
\end{array}\right\}
$$

or:

$$
L(\hat{x})(y-\hat{y})=\left\{\begin{array}{c}
L_{1}\left(x_{1}-\hat{x}_{1}\right)+L_{3}\left(x_{3}-\hat{x}_{3}\right)+L_{5}\left(x_{5}-\hat{x}_{5}\right) \\
0 \\
L_{7}\left(x_{1}-\hat{x}_{1}\right)+L_{9}\left(x_{3}-\hat{x}_{3}\right)+L_{11}\left(x_{5}-\hat{x}_{5}\right) \\
0 \\
L_{13}\left(x_{1}-\hat{x}_{1}\right)+L_{15}\left(x_{3}-\hat{x}_{3}\right)+L_{17}\left(x_{5}-\hat{x}_{5}\right) \\
0
\end{array}\right\}
$$

A pair of state dependent matrices $A(x)$ and $B(x)$ is chosen among several possibilities for this problem. In the SDRE technique matrices $A(x)$ and $B(x)$ are not unique and one cannot guarantee that this or that specific choice of these matrices will produce the best (optimal) results. In this sense, the problem investigated and discussed here is a suboptimal problem. 


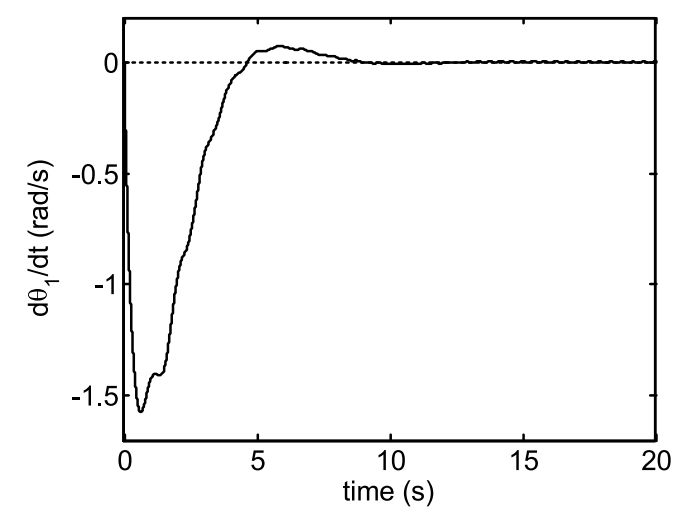

Fig. 9. Estimated behavior of variable $\dot{\theta}_{1}$ considering same initial conditions for the angular displacements.

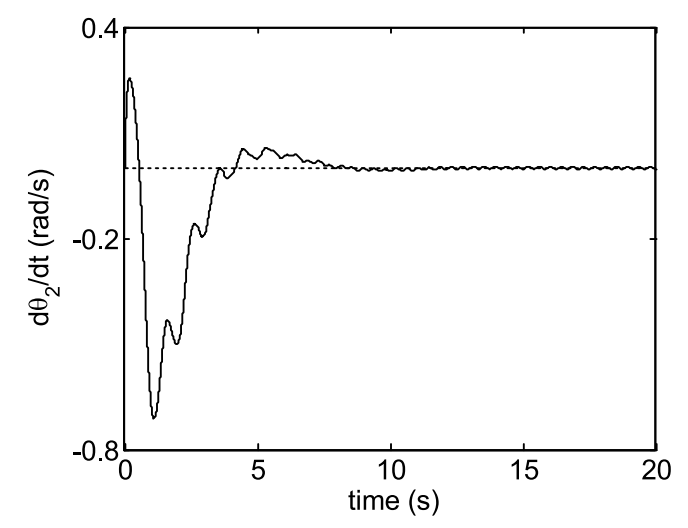

Fig. 11. Estimated behavior of variable $\dot{\theta}_{2}$ considering same initial conditions for the angular displacements.

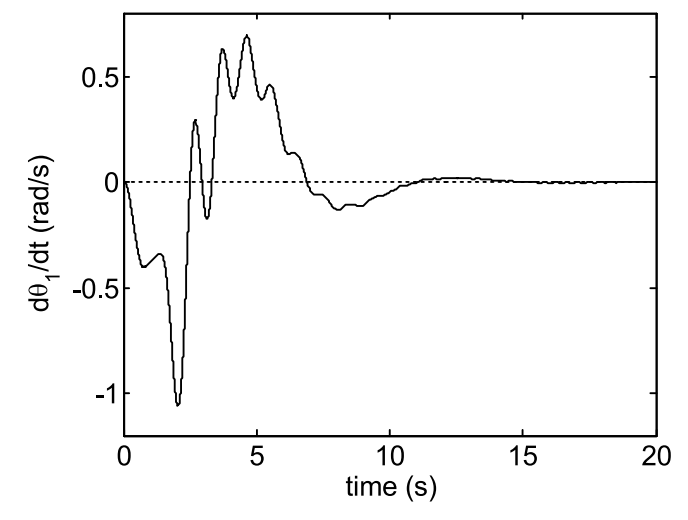

Fig. 10. Estimated behavior of variable $\dot{\theta}_{1}$ considering different initial conditions for the angular displacements.

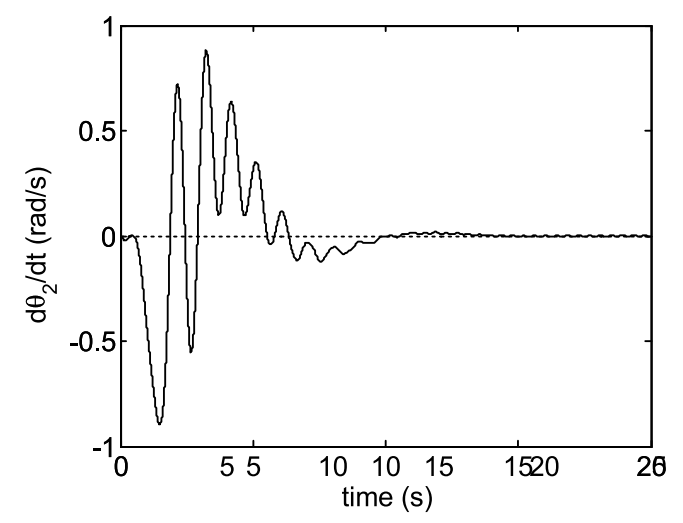

Fig. 12. Estimated behavior of variable $\dot{\theta}_{2}$ considering different initial conditions for the angular displacements.

The time behavior of angle $\theta_{1}$ is presented in Figs 3 and 4. In Fig. 3 the same initial condition is considered for the "real" system and for the estimator. In Fig. 4 different initial conditions are considered for the "real" system and for the estimator. The different initial conditions are chosen in order to consider a situation in which the error between "real" and estimated is sufficiently great and the nonlinearities are sufficiently excited.

The same idea is illustrated in Figs 5 and 6 for the angular variable $\theta_{2}$ and in Figs 7 and 8 for the time component of the variable of deflection, $q_{1}$.

The time behavior of estimated velocity $\dot{\theta}_{1}$ is presented in Figs 9 and 10. In Fig. 8 the same initial condition for the angular displacements is considered for the "real" system and for the estimator. In Fig. 10 different initial conditions for the angular displacements are considered for the "real" system and for the estimator. The same idea is presented in Figs 11 and 12 for the estimated velocity $\dot{\theta}_{2}$ and in Figs 13 and 14 for the estimated velocity $\dot{q}_{1}$.

The control torque $Q_{\theta 1}$ is presented in Fig. 15. In this figure, the curve considering same initial condition for "real" and estimated angular displacements is compared with a curve that considers different initial conditions for "real" and estimated angular displacements. The same idea is presented in Fig. 16 for the control torque $Q_{\theta 2}$.

As depicted in Figs 3 to 6, the angles $\theta_{1}$ and $\theta_{2}$ are satisfactorily controlled considering both the same and different initial conditions between "real" and estimated systems. In all the cases considered the angles reach the desired final states. The same is true for the variable $q_{1}$ in Figs 7 and 8 since the amplitudes of the beam vibration also go to zero with time. The influence of the not controlled variable $q_{2}$ is noticed in these figures as the time decaying vibration in steady state.

All the estimated velocities presented in Figs 9 to 14 are consistent with the system dynamics and were used without problem for control. The control torques are depicted in Figs 15 and 16. 


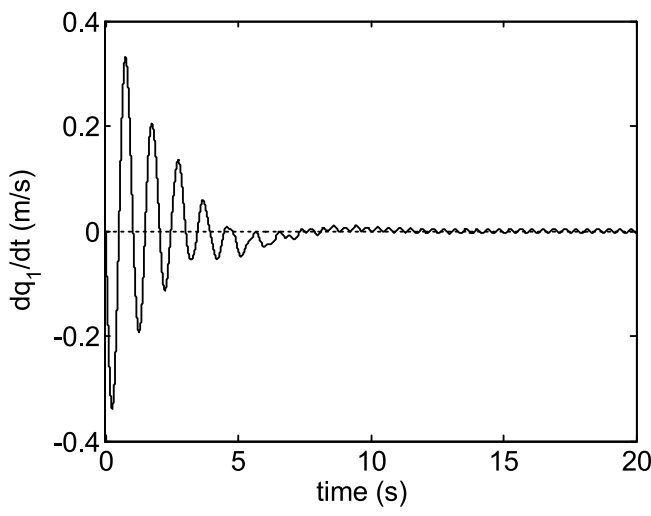

Fig. 13. Estimated behavior of variable $\dot{q}_{1}$ considering same initial conditions for the angular displacements.

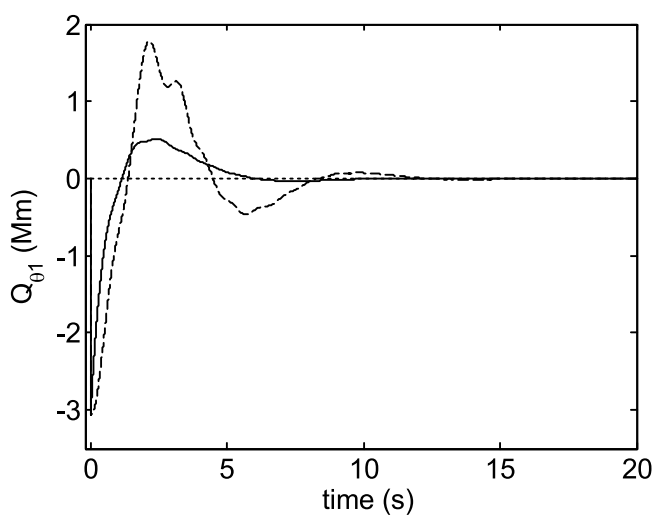

Fig. 15. Control torque $Q_{\theta 1}$ considering same initial conditions (-) and different initial conditions (- - ) for the angular displacements.

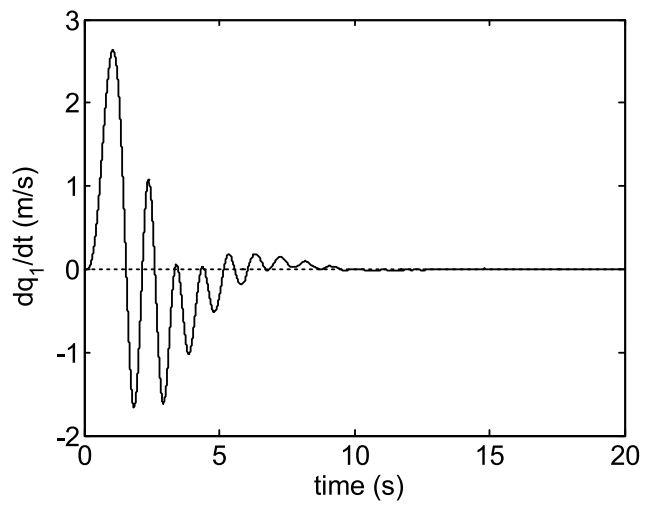

Fig. 14. Estimated behavior of variable $\dot{q}_{1}$ considering different initial conditions for the angular displacements.

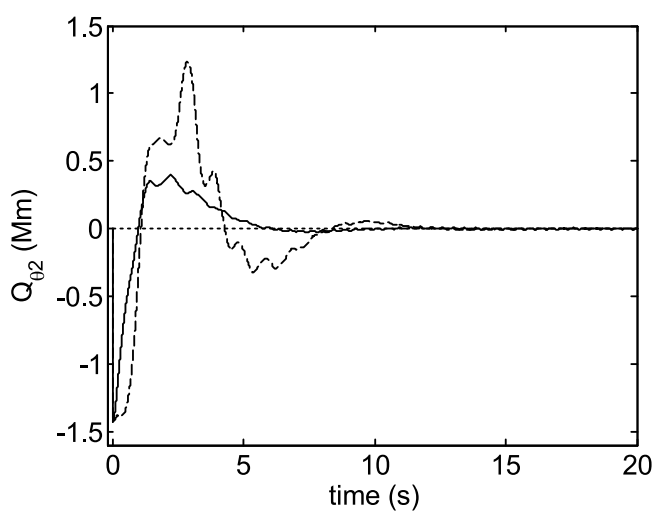

Fig. 16. Control torque $Q_{\theta 2}$ considering same initial conditions (-) and different initial conditions (- - ) for the angular displacements.

\section{Discussion and conclusions}

In the models investigated in this work, no friction forces or structural damping is considered. The dissipating effects related to these influences will be considered further on this research since all real systems presents in some degree influences due these effects. In this sense, in all the results presented here, all the amplitudes attenuation illustrated in the figures are due to the nonlinear control actuation only. One problem still to be solved in this investigation is the overshoot in the initial time of the simulations.

The results obtained using the nonlinear control technique named SDRE and the nonlinear state estimation procedure based on this same technique for the rigid-flexible two link robot manipulator are completely satisfactory in the sense that the angular displacements of both slewing axes converge to the desired final values and at the same time the vibration on the beam (first mode) is eliminated. It was considered large angular displacements and velocities and the nonlinear terms present in the governing equations of motion are sufficiently important in the cases studied here.

For initial estimated states near the initial "real" states the error between "real" and estimated responses are negligible. For initial estimated states and initial "real" states sufficiently distant from each other the estimated states converges to the "real" (measured) states in a satisfactory way.

The above conclusions are true even when considering two different mathematical models: one emulating the "real" system (considering two modes expansion for $v(x, t)$ ) and one different model representing a simpler mathematical model the "real" system and used for estimation (considering one modes expansion for $v(x, t)$ ). The second 
mode present in the "real" model is neither "measured" nor estimated and therefore not controlled. Its influence can be slightly noted in the response of the system as a small amplitude decaying vibration.

The SDRE method proposed to tune the observer gains is used here because it utilizes the same structure of the nonlinear control technique with the same name (SDRE) and intended to be tested in this work. In further developments, other nonlinear state estimation techniques must be tested and compared with the results presented here in order to provide the best method.

One major problem verified in the numerical simulations shown here is the presence of overshoot in the time responses. The elimination of this overshoot is the next step in the improvement of this research.

\section{References}

[1] E. Barbieri and Ü. Özgüner, Unconstrained and constrained mode expansions for a flexible slewing link, Transactions of the ASME 110 (December 1988), 416-421.

[2] R.W. Clough and J. Penzien, Dynamics of structures, McGraw-Hill, Tokio, Japan, 1975.

[3] J.R. Cloutier, D.T. Stansbery and M. Sznaier, On the recoverability of nonlinear state feedback laws by extended linearization control techniques, Proceedings of the American Control Conference, San Diego, California (June 1999), 1515-1519.

[4] T. Çimen, State-dependent riccati equation (SDRE): A survey, Proceedings of the $17^{\text {th }}$ World Congress, The International Federation of Automatic Control, Seoul, Korea (6-11 July 2008), 3761-3775.

[5] T. Çimen and A.O. Merttopçuoğlu, Asymptotically optimal nonlinear filtering: Theory and examples with application to target state estimation, Proceedings of the 17th World Congress of The International Federation of Automatic Control, Seoul, Korea (6-11 July 2008).

[6] A. Fenili, Mathematical modeling and analysis of the ideal and nonideal behavior of slewing flexible structures, Ph.D Thesis, University of Campinas (UNICAMP), Faculty of Mechanical Engineering, Brazil, 2000, In Portuguese.

[7] A. Fenili, Position and vibration control of a nonlinear slewing flexible structure using the sdre method, 8th Brazilian Conference on Dynamics, Control and Applications - Dincon 2009/18 A 22 DE MAIO DE 2009 - Bauru, SP/Brasil.

[8] A. Fenili and J.M. Balthazar, Control of a rigid-flexible two link nonlinear robotic manipulator using the SDRE method, 10th Conference on Dynamical Systems Theory and Applications, Łódź, Poland (7-10 December 2009).

[9] A. Fenili and J.M. Balthazar, The rigid-flexible nonlinear robotic manipulator: Modeling and control, Communications in Nonlinear Science and Numerical Simulations 16(5) (May 2011), 2332-2341.

[10] E. Garcia, On the modeling and control of slewing flexible structures, Ph.D Thesis, Faculty of the Graduate School of the State University of New York at Buffalo, 1989.

[11] D.A. Haessig and B. Friedland, State dependent differential riccati equation for nonlinear estimation and control, 15th IFAC Triennial World Congress, Barcelona, Spain (July 2002).

[12] R.A. Hull, J.R. Cloutier, C.P. Mracek and D.T. Stansbery, State-dependent riccati equation solution of the toy nonlinear optimal control problem, Proceedings of the American Control Conference, Philadelphia, Pennsylvania (June 1998), 1658-1662.

[13] J.M. Renno, Inverse dynamics based tuning of a fuzzy logic controller for a single-link flexible manipulator, Journal of Vibration and Control 13 (2007), 1741, DOI: 10.1177/1077546307076282.

[14] R.M. Rosenberg, Analytical dynamics of discrete systems, Plenum Press, New York, 1977.

[15] J.J. Sah and R.W. Mayne, Modeling of a slewing motor-beam system, Proceedings of the International Computers in Engineering Conference, Boston (1990), 481-486.

[16] J.S. Shamma and J.R. Cloutier, Existence of SDRE stabilizing feedback, IEEE Transactions on Automatic Control 48 (2003), $513-517$.

[17] M.O. Tokhi and A.K.M. Azad, Flexible robot manipulators: Modelling, simulation and control, Published by The Institution of Engineering and Technology, London, UK, 2008. 

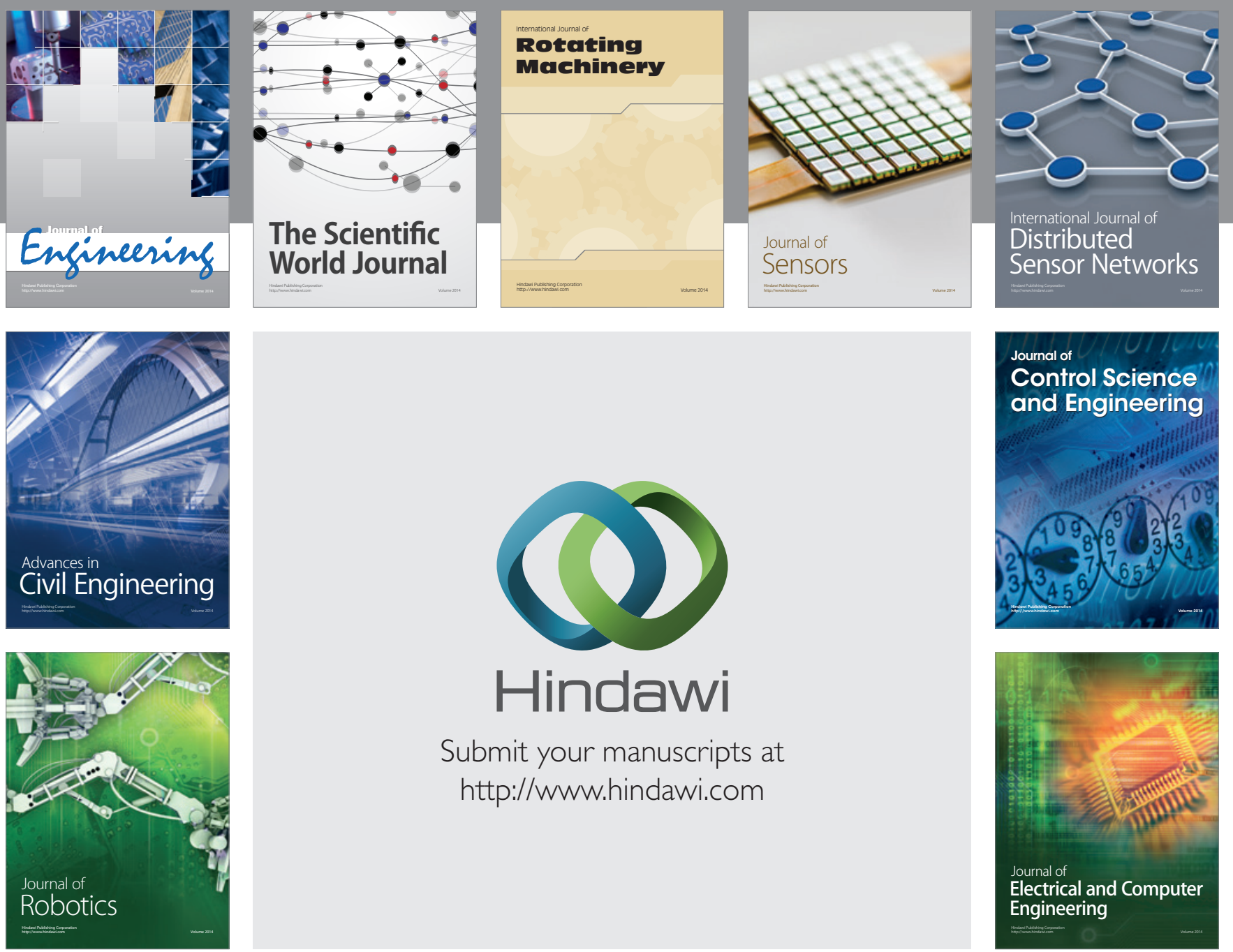

Submit your manuscripts at

http://www.hindawi.com
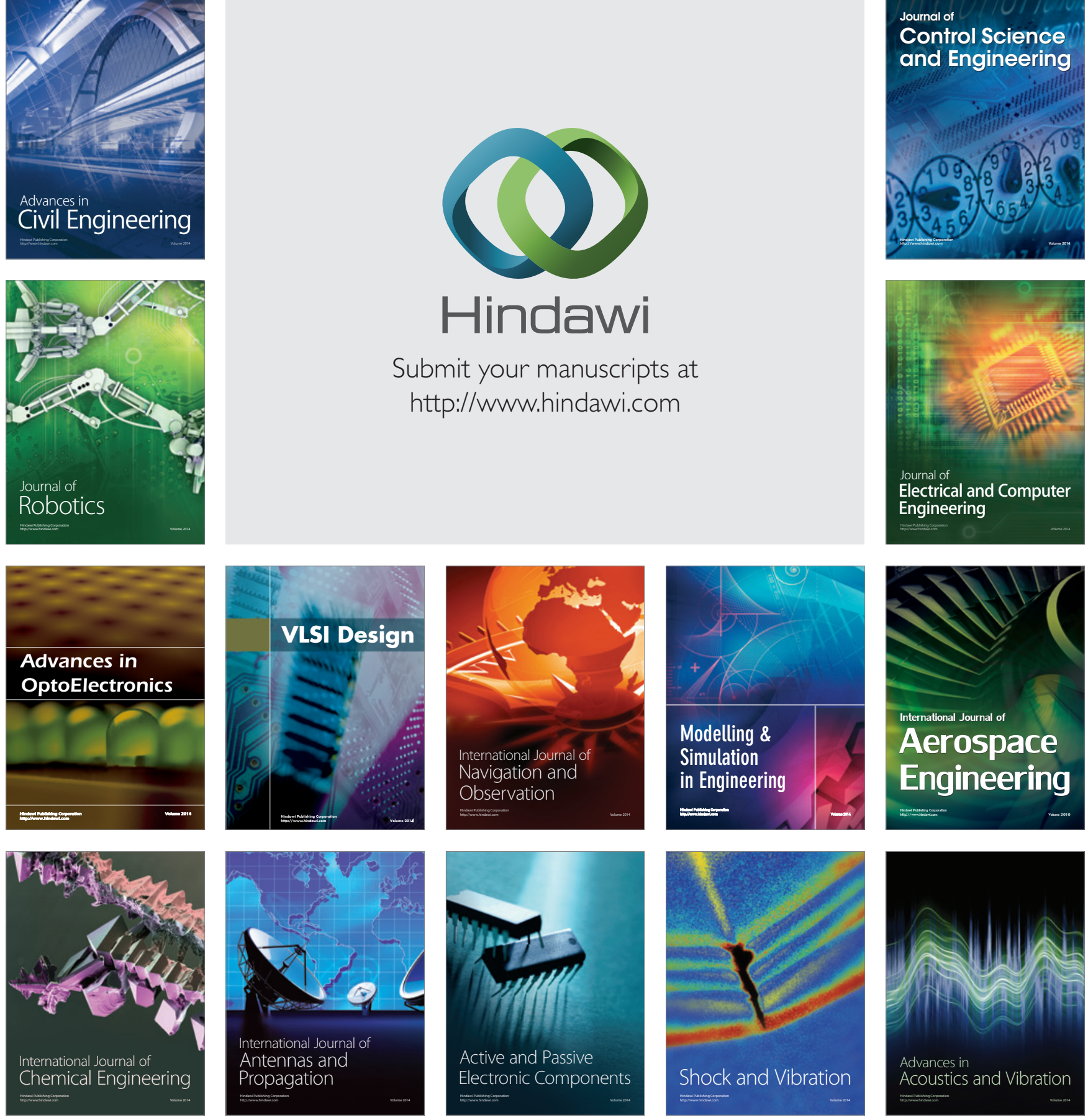